\title{
Werner Rügemer
}

\section{Global Corruption}

In der bürgerlichen Wirtschaftswissenschaft wird Korruption als nicht existent betrachtet, in globalisierungskritischer Darstellung und marxistischer Analyse wird sie bisher achtlos vernachlässigt. In Politik und Medien erscheint Korruption als jeweils skandalöser Einzelfall. Das hängt mit der lobend oder kritisch gemeinten Auffassung zusammen, der gegenwärtige Kapitalismus und dessen neoliberale Ausgestaltung stellten „die Herrschaft des Marktes" dar. So harmlos ist die Sache jedoch keineswegs. Die „unsichtbare Hand“ des Marktes - die angeblich von selbst Angebot und Nachfrage regelt - war und ist organisch verbunden mit anderen Instrumenten, und zwar nicht nur mit der sichtbaren militärischen Faust, die periodisch zur Marktexpansion und Marktsicherung eingesetzt wird. Der "Markt" funktioniert nirgends nach der Lehrbuchdoktrin vom "freien Spiel der Kräfte". Neben die selektive Staatsgewalt und den Einsatz militärischer Mittel tritt die Korruption. Sie gehört zum systemischen Instrumentarium der „unsichtbaren Hand“ der „Marktwirtschaft" in den Kapitaldemokratien. Dies gilt in verstärktem Masse für deren neoliberale Phasen. Der gegenwärtige neoliberale Globalismus beinhaltet die bisher weitestgehende, nachhaltige Entfesselung der Korruption in der Geschichte. Korruption wird hier ständig modernisiert und legalisiert und entkommt in den meisten Fällen der öffentlichen Wahrnehmung und Inkriminierung. Globale Gestaltungsformen korruptiver Geldflüsse eilen den national begrenzt gehaltenen Möglichkeiten der Justiz davon. Gleichzeitig ist die Sensibilität gewachsen: Internationale Organisationen, nationale Parlamente und Unternehmensleitungen haben die Bekämpfung der Korruption zum festen Ziel erklärt. Doch ohne den Aufstand der Zivilgesellschaft von innen und von unten kann sich in Richtung substanzieller Demokratie nichts wenden.

\section{Das "System Elf"}

Lange Zeit trugen die Entwicklungsländer das Brandzeichen der Korruption. Diktatoren wie Marcos (Philippinen), Mobutu (Kongo), Schah Pahlevi (Iran), Duvalier (Haiti), Noriega (Panama), Bongo (Gabun), Roh (Südkorea) und zuletzt beispielsweise Abacha (Nigeria) gaben dieser Wahrnehmung immer neue Nahrung. Dabei richtete sich die öffentliche Aufmerksamkeit einseitig auf die 
Empfänger der Bestechungsleistungen. Erst allmählich wird die banale, aber grundlegende Tatsache wahrgenommen, dass zur Korruption zwei Akteure gehören: Geber und Empfänger, Bestecher und Bestochener. Die Diktatoren der Entwicklungsländer sind nur die eine Seite einer korruptiven Gemeinschaft; die andere Seite sind die Geber aus den „entwickelten“ Industriestaaten (Pieth/Eigen 1999).

Gegenwärtig ermöglicht das Gerichtsverfahren um den ehemaligen französischen Staatskonzern Elf-Aquitaine einen tiefen Einblick in Tradition und Strukturen globaler Korruption. Das Verfahren mit 37 Angeklagten hat am 17. März 2003 begonnen und soll vier Monate dauern. Es ist das umfangreichste gerichtliche Korruptionsverfahren, das je in einem Staat der „westlichen Wertegemeinschaft" stattgefunden hat. Staatsanwälte und Kriminalbeamte haben ein Jahrzehnt aufwendig ermittelt, sie waren Intrigen ausgesetzt und konnten nur unter Polizeischutz ihrer Arbeit nachgehen. Die Unterlagen kamen auch durch Amtshilfe aus anderen Ländern zusammen, so aus der Schweiz, aus Monaco, Italien, Luxemburg und aus Afrika.

Angeklagt sind der ehemalige Vorstandsvorsitzende Le Floch-Prigent, weitere Vorstandsmitglieder, ehemalige Minister, Staatssekretäre, Mitglieder von Parteivorständen, hochrangige Geschäftsleute. Sie sind heute meist als Unternehmensberater tätig. Sie waren oder sind Mitglieder der politisch-wirtschaftlichen Elite, sie nennen vor Gericht ihre Orden und staatlichen Auszeichnungen, darunter die Légion d'honneur. Als aktuelle Wohnsitze geben sie Monaco, Paris, London, Zürich und Moskau an. Ihre 80 Anwälte gehören zu den teuersten Frankreichs. Die Endempfänger im Ausland stehen nicht vor Gericht.

In den Jahren zuvor fanden bereits Einzelverfahren statt. Unabhängig von den Ergebnissen des jetzigen Verfahrens stehen die Eckdaten des „Systems Elf" fest (vgl. Georgel/Thorel 1997: 73ff). Aus „schwarzen Kassen“ des Konzerns wurden jahrzehntelang verdeckte Zahlungen geleistet. Sie flossen einmal an Politiker und Beamte der Staaten, in denen es für Elf um Öl-Förderrechte oder andere Geschäfte im Zusammenhang mit Erdöl ging. Dauerempfänger von Millionenzahlungen waren etwa der schon genannte Präsident von Gabun, Bongo, sowie die Präsidenten N'Guesso (Kongo-Brazzaville) und Biya (Kamerun). Eine zweite Empfängergruppe waren führende Politiker der Regierungsparteien in Frankreich selbst, darunter die des gegenwärtigen Präsidenten Chirac, sowie deren Präsidentschaftskandidaten. Die Zahl der Geber, Empfänger, mitverdienenden Vermittler und ihrer Helfer beträgt mehrere hundert.

Die Gesamtsumme der für das Verfahren herangezogenen Zahlungen beträgt 430 Millionen Euro (Financial Times 18.3.2003). Ihr tatsächlicher Umfang ist jedoch weit höher. Wegen der Verjährungsfristen und der Ermittlungsbedingungen geht es nur um die Jahre 1990 bis 1995. Darüber hinaus wurden mehrere Teilkomplexe ausgeklammert, so die 35 Millionen Euro, die von Elf für den günstigen Kauf der ostdeutschen Minol-Tankstellen und des Chemie- 
komplexes Leuna in Sachsen-Anhalt „in Richtung“ deutscher Parteien gezahlt wurden, wo die Endempfänger aber bis heute unbekannt blieben (vgl. dazu Leyendecker u.a. 2000). Ausgeklammert ist auch etwa der Fall des Finanziers Auchi, der 41 Millionen Euro für seine Vermittlung beim Kauf des spanischen Ölunternehmens Cepsa Ertoil erhalten haben soll - die Londoner Behörden haben den Geschäftsmann mit britischem Pass nicht ausgeliefert.

Zum „System Elf ${ }^{\text {‘s }}$ gehört die verdeckte Lenkung der Geldflüsse. Die dem Vorstand zugeordneten Berater Sirven und Tarallo hatten die Verwaltung der schwarzen Kassen unter sich. Sie wurden in mehreren Briefkastenfirmen in der Schweiz bewirtschaftet. Afrikanische Staatschefs ebenso wie französische Parteien unterhielten in der Schweiz für den Empfang ebenfalls Briefkastenfirmen. Die Gelder wurden intern wechselweise als "geheime Fonds“, als „Interventionsfonds" oder schlicht als „Fonds" oder „Kommissionen" bezeichnet (Le Monde 1.4.2003).

Zum „System Elf" gehört weiter die Selbstbereicherung des Topmanagements. Die Zahlungen gingen nicht vollständig an die Endempfänger. Einen Anteil behielten Topmanager und Berater für sich - Beträge in zweistelliger Millionenhöhe. Der Vorstandsvorsitzende konnte über weitere Beträge verfügen, etwa wenn er einer Geliebten lukrative Aufträge verschaffte oder wenn er aus einer der schwarzen Kassen Kredite an ein Textilunternehmen gewährte, mit dessen Inhaber er befreundet war.

Beim „System Elf" wird schließlich deutlich, dass eine große Zahl hochrangiger Akteure, Förderer und Mitwisser erforderlich ist. Die Buchführung des Konzerns ebenso wie die Wirtschaftsprüfer waren an der professionellen Gestaltung des mehrfach illegalen Geldflusses beteiligt. Sie sorgten durch Scheinrechnungen von ausländischen Tochterfirmen dafür, dass die schwarzen Kassen gefüllt wurden. Der Staat akzeptierte die Geheimoperationen als rechtmäßig. Gegenüber dem Finanzamt wurden die Kommissionen als „Bonuszahlungen", „Abonnements" oder auch als „vorgezogene Anerkennungszahlungen bezeichnet und als solche steuerbegünstigt anerkannt.

Damit erreichte der Konzern, dass er Öl zu so günstigen Konditionen erhielt, wie es wettbewerbsmäßig nicht möglich gewesen wäre. Französische Politiker deckten die Praktiken und sorgten durch ihre Komplizenschaft für die „richtige" Besetzung der Posten im Topmanagement. Elf erlangte durch die dauerhafte Abhängigkeit von Politikern und Beamten einen Extraprofit. Auf der anderen Seite trug der Staatskonzern zur Unterhöhlung und Zerstörung der Demokratie in mehreren Staaten bei. In Frankreich bildete sich zwischen der Unternehmensspitze und den Staats- und Parteispitzen ein elitärer, geheimdienstgeschützter, „Staat im Staat".

Elf konnte sich darauf berufen, dass man zu solchem Verhalten gezwungen sei, da die internationalen Konkurrenten genauso vorgehen. Dies stellt einen weiteren Hinweis auf den systemischen Charakter der Korruption dar. Ob- 
wohl es sich bei Elf Aquitaine um einen Staatskonzern handelte - er wurde erst im Jahre 2000 privatisiert und von Totalfina aufgekauft -, weicht seine Korruptionspraxis kaum von der Praxis der Mitkonkurrenten und anderer privater Großunternehmen ab. Die Vorratshaltung von schwarzen Kassen in spezialisierten Tochterfirmen, die Einschaltung von staatlichen Geheimdiensten, die Nutzung von Finanzoasen, der Einsatz von Beratern und Vermittlern, die parteiübergreifende Bestechung von Politikern im In- und Ausland solche Systeme sind seit Jahrzehnten in den internationalen Geschäftsbeziehungen üblich.'

Dies wurde, um zunächst in Frankreich zu bleiben, bei Gerichtsverfahren etwa gegen den größten Baukonzern der Welt, Bouygues, bestätigt. Er zahlte beispielsweise 900 Millionen Dollar Kommissionen für den 6-Milliarden-DollarAuftrag des Baus der Universität Riad (Saudi-Arabien). Allerdings ist auch eine Modernisierung der Mittel festzustellen. Es muss schon längst nicht immer Bargeld sein: nachdem Bouygues Anteile am größten französischen TV-Sender erworben hatte, wurde das „Prinzip Bouygues" entwickelt: So erhielt die Tochterfirma SAUR des Baukonzerns als Gegenleistung für eine Gefälligkeitsreportage über den Präsidenten Houphouet-Boigny (Elfenbeinküste) die Lizenz zum Betreiben von Wasseranlagen in dem afrikanischen Land. Für eine Sendung voller Lobeshymnen über König Hassan von Marokko erhielt die Tochterfirma Bouygues Batiment den Auftrag für den Flughafen von Agadir (Raffy 1996).

Ähnliche globale Korruptionssysteme wie bei Elf-Aquitaine und Bouygues wurden bei Konzernen verschiedener Staaten offengelegt. Auch hier stellte sich heraus, dass multinationale Konzerne dies seit Jahrzehnten praktizieren, in Italien (Calvi/Sisti 1995), der Schweiz (Queloz u.a. 2000), den USA (Jacoby/Nehemkis/Eells 1977), Saudi-Arabien (Aburish 1995) und beim „Exportweltmeister" Deutschland (Rügemer 1996: 17ff). Projektbereiche, in denen immer wieder Korruption aufgedeckt wird, betreffen neben dem Rüstungsund Industrieanlagengeschäft den Bau von Staudämmen, Kraftwerken, StraRen, Autobahnen, U-Bahnsystemen, Immobilien, Wohnsiedlungen, Zulieferungen und die Bestückung von Marktketten. Dies trifft in verstärktem Masse zu, wenn es gleichzeitig um Privatisierungen und langfristige Wartungs- und Entsorgungsverträge geht. ${ }^{2}$

1 Vgl. Colombo (1995: 41), Rügemer (1996: 18ff) hier werden auch die nationalen Korruptionsprofile Deutschlands, der USA, Frankreichs, Saudi-Arabiens, Südkoreas, Indiens, Chinas und der postsozialistischen Staaten dargestellt.

2 Vgl. die laufende Berichterstattung in den von Transparency International herausgegebenen nationalen und internationalen. Newsletters und im jährlichen Global Corruption Report. 


\section{Korruption in den kapitalistischen Zentren}

Nun könnte der Eindruck aufkommen, dass die globale Korruption vor allem darin besteht, dass Geber aus den kapitalistischen Hauptländern und Empfänger aus Entwicklungsländern aufeinandertreffen. Wie aber schon angedeutet, gehört zum exemplarisch vorgestellten „System Elf" auch die Bestechung von Politikern und Beamten im Stammland eines Konzerns, hier in Frankreich. Die innerhalb der kapitalistischen Hauptländer gezahlten Korruptionssummen erreichen eine wesentlich größere Dimension als die vielfach spektakulär dargestellten Zahlungen in die Entwicklungsländer. Das liegt darin begründet, dass in den Industriestaaten die Ansprüche der Politiker, Beamten und weiterer Entscheidungsträger aufgrund des höheren Einkommensniveaus naturgemäß höher sind; zweitens sind an einer Auftragserteilung von Großprojekten in einem Rechtsstaat bis zu zwei Dutzend Personen beteiligt, die je nach Entscheidungskompetenz und hierarchischer Stellung ihren Anteil erhalten. Drittens ist das Gesamtvolumen der Aufträge in einer einzigen europäischen oder US-amerikanischen Großstadt größer als in manchem Entwicklungsland. So ist allein das Bauvolumen der Müllverbrennungsanlage der Stadt Köln genauso hoch wie das des größten Bauprojekts in Uganda (mehr dazu unten). Die jeweils nicht neu verhandelten, sondern professionell feststehenden Schmiergeldanteile liegen zwischen drei und sieben Prozent, können aber in besonders umkämpften und dynamischen Bereichen - etwa bei der Einführung neuer Medikamente, neuer Softwaresysteme und Computer - ebenso wie in extrem monopolistischen Märkten wie der Rüstung weit darüber liegen.

Deutsche Konzerne, die international wegen Korruption auffällig geworden sind, werden in der Regel auch in Deutschland auffällig. Das gilt etwa für den Siemens-Konzern. Ob Griechenland, Uruguay, Südkorea oder Singapur: Siemens ist dabei, aber eben auch in Deutschland selbst. Ein Großverfahren gegen fünf Siemens-Manager wegen Bestechung für einen Klärwerksauftrag in München machte deutlich, dass hier mit denselben Methoden gearbeitet wird wie in Entwicklungsländern: Einschaltung eines externen Vermittlers, verdeckter Geldfluss über eine Finanzoase, Einrichtung eines Nummernkontos für den Empfänger in der Schweiz, Falschdeklarierung der Kommissionszahlung, Überhöhung des Auftragspreises. Die hochrangigen Manager, die zu Gefängnis- und Geldstrafen verurteilt wurden, zeigten kein Schuldbewusstsein. Das Unternehmen Siemens übernahm alle Anwaltskosten und gewährte den Verurteilten auch während ihres Gefängnisaufenthalts nicht nur ihr reguläres Gehalt, sondern auch die ihnen sonst zustehenden Gehaltserhöhungen (Rügemer 1995: 139ff). Korruption liegt im Firmeninteresse und ist normaler Bestandteil des Managementhandelns.

Korruption ist in den deutschen Kommunen traditionell tief verankert (vgl. Bannenberg 2002, Rose 2003, Rügemer 2002, Heußner 2002). Das zeigt sich 
bei den wiederkehrenden Bauskandalen, zuletzt in größerem Ausmaß beim Bau von Müllverbrennungsanlagen in Köln, Böblingen und einem weiteren Dutzend Städten. Hier verhielt sich etwa der Generalunternehmer Steinmüller in Deutschland wie in Südafrika. Dort hatte Steinmüller zusammen mit anderen deutschen Unternehmen wie der Deutschen Bank und Daimler jahrelang die Apartheidpartei National Party heimlich finanziert, um an Aufträge für den Bau von Bergwerksanlagen zu kommen. In Köln und weiteren deutschen Städten wie Böblingen zahlte Steinmüller an örtliche Politiker und Beamte, um den Auftrag für die Müllverbrennungsanlagen zu bekommen. In Köln betrug der Inhalt des Schmiergeldtopfes, der juristisch seinen Standort in der Schweiz hatte, 15 Millionen Euro, die an ein knappes Dutzend Empfänger ausgezahlt wurden. Beim Bau der deutschen Müllöfen wurde der reguläre demokratische Entscheidungsprozess ausgehebelt ebenso wie in Südafrika ein undemokratisches System gestützt wurde.

Der Preis der Kölner Anlage stieg zugunsten des Bestechers weit über den Marktpreis, die Anlage wurde heimlich weit größer gebaut als genehmigt, die Müllgebühren stiegen nach der Fertigstellung stärker als angekündigt. Ein europaweiter, bis nach Neapel reichender Mülltourismus wurde in gang gesetzt, um die überdimensionierte Kölner Anlage zu füllen (Rügemer 2002: 11ff). Das gleiche grenzüberschreitende Korruptionsverhalten zeigten die Schweizer Anlagenbaufirmen $\mathrm{ABB}$ und von Roll, die in mehreren deutschen und luxemburgischen Städten Kommunalpolitiker bestachen. Übrigens wurden, wie bei Großprojekten häufig der Fall, die meisten Schmiergelder über ein Ingenieurbüro eingesammelt und weitergeleitet, ein Verfahren, das auch in Entwicklungsländern praktiziert wird (Der Stern 13/2002: 28ff, Carini 2002).

Wenn man die Tatsache berücksichtigt, dass zur korruptiven Gemeinschaft zwei Akteure gehören, dann fallen beliebte Klischees wie „Italien ist ein korruptes Land" in sich zusammen. So hatten die deutschen Pharmakonzerne Bayer und Boehringer seit den 70er Jahren in Italien zwischen Palermo und Mailand Labor- und Krankenhauspersonal bestochen, um den Verkauf von Medikamenten und Wirkstoffen zu überhöhten Preisen abzusichern. Ähnlich gingen zur gleichen Zeit die angloamerikanischen Pharmaunternehmen Beckman und Dasit in Italien vor (Rügemer 1996: 39). Wenn der eine Akteur desselben Korruptionsvorgangs aus Italien kommt, der andere jedoch aus Deutschland, Großbritannien oder den USA, so lässt sich das Klischee „Italien ist ein korruptes Land" nicht mehr aufrechterhalten.

Gegenwärtig wird in Deutschland der „Herzklappenskandal“ gerichtlich abgearbeitet. Die zwei US-amerikanischen Hersteller medizinischer Geräte, St. Jude Medical und Medtronic, hatten Ärzte mithilfe verschiedener Vorteilsgaben dazu gebracht, Herzklappen und anderes Material der Herzchirurgie exklusiv bei diesen Firmen einzukaufen, und zwar zu Preisen, die bis zu 100 Prozent überteuert waren. Für jede einzelne Herzklappe erhielten die Ärzte einen „Er- 
folgsbonus“ von einigen hundert Euro. Entsprechend dem Klischee „Italien ist ein korruptes Land“ müsste man nun sagen: „Deutschland ist ein korruptes Land“. Dies wäre zwar genauso wenig falsch wie bezüglich Italien, aber man müsste dann auch sagen: „Die USA sind ein korruptes Land“, denn von dort operierten in diesem Fall, zudem straflos, die Bestecher. Aber die Aussage, es gebe bestimmte Länder, die besonders korrupt seien, während andere es weniger sind, ergibt keinen analytischen Sinn, denn dabei wird nur die Empfängerseite berücksichtigt.

\section{Legalisierte Korruption}

Die modernen bürgerlichen Kapitaldemokatien haben in ihren Aufstiegsphasen Korruption strafrechtlich inkriminiert. Dabei ging es vor allem um das Schutzgut "Ansehen des Staates“. Deshalb wurde vor allem der "Staatsdiener“ unter Strafe gestellt, wenn er gegen Vorteilsgewährung eines Dritten staatliche Vorschriften und Gesetze verletzte. So wurde es beispielsweise im Bürgerlichen Gesetzbuch des Deutschen Reiches Ende des 19. Jahrhunderts geregelt und gilt im wesentlichen bis heute.

Aber schon nach dem ersten Weltkrieg gingen die entwickelten Industriestaaten dazu über, die von den Unternehmen gezahlten Schmiergelder steuerlich zu fördern, das betraf auch solche an Staatsdiener selbst. Korruptionsleistungen zur Geschäfts- und Vertragsanbahnung wurden als „nützliche Ausgaben“ betrachtet und als steuermindernde Betriebsausgaben anerkannt. In Deutschland geschah dies im ersten Jahressteuergesetz 1934 des NS-Regimes, das mit einem Programm der konsequenten Korruptionsbekämpfung angetreten war (Rügemer 1996: 60).

In Deutschland bedeutete das NS-Regime überhaupt einen Schub für die professionelle Unternehmenskorruption. Mit staatlicher Hilfe, insbesondere des „Vier-Jahres-Plans" unter Göring, wurden von deutschen Unternehmen und Banken insbesondere in der Schweiz, in Monaco und in der US-Finanzoase Delaware Hunderte von Briefkastenfirmen eingerichtet, um Schmiergelder für wichtige Material- und Devisenbeschaffungen verdeckt an die Empfänger zu leiten (Rügemer 2002: 130ff). Nach dem zweiten Weltkrieg wurden solche Praktiken in der Exportwirtschaft ausgeweitet und normalisiert. Auch die geheime finanzielle Ausstattung der „wirtschaftsfreundlichen“ Parteien CDU, CSU und FDP durch den Bundesverband der Deutschen Industrie und durch Hunderte von Unternehmen wurde so organisiert. Etwa 220 Millionen DM flossen auf diesen Wegen straflos in die Kassen staatstragender Parteien (Kilz 1983). Es ist deshalb kaum erstaunlich, dass Korruptionsleistungen nach dem zweiten Weltkrieg auch für die globale Auftragsakquisition der Unternehmen staatlich gefördert wurden. Dies geschah offiziell und gesetzlich, aber der Öffentlichkeit weitgehend verschwiegen, durch die steuerliche Begünstigung als 
„nützliche Ausgaben“ und durch die staatliche Exportversicherung („HermesKredite").

So wurde in den konfligierenden Wertsetzungen - strafrechtliche Verfolgung der Korruption bei den Staatsdienern einerseits, Förderung der Unternehmenskorruption für die Geschäftsanbahnung im In- und Ausland andererseits - die Seite der Geschäftsanbahnung immer stärker wirksam. Dies war noch weitergehend der Fall, je mehr sich die neoliberale Auffassung durchsetzte, dass auch der Staat und staatliche Behörden sich selbst als Unternehmen, ja als „profit center" zu verstehen hätten. Dies hatte zur Folge, dass einerseits die strafrechtliche Definition und Verfolgung auf einem nostalgisch-anachronistischen Stand verblieb, der sich in Deutschland vor allem in den Strafrechtsparagraphen 331 bis 334 manifestiert. Andererseits wurde Korruption so modernisiert, dass die meisten wichtigen Korruptionsformen heute nicht unter Strafe stehen. Sie sind als „legalisierte Korruption“ zu bezeichnen.

Dazu zählen die heute vorherrschenden Formen, mit deren Hilfe Unternehmen Politiker und Staatsdiener in Abhängigkeit bringen. Dabei herrscht nicht mehr das alte Prinzip, dass auf einen gewährten Vorteil (Bargeld, geldwerte Leistung) unmittelbar die vereinbarte Gegenleistung folgt (staatlicher Auftrag). Vielmehr handelt es sich um eine langfristige Netzwerkbildung, bei der Leistung und Gegenleistung zeitlich und operativ entzerrt sind: Unbefristete Beraterverträge mit regelmäßigen monatlichen Zahlungen, Mitgliedschaften in Aufsichtsräten und Arbeitsverträge ohne entsprechende Arbeitsleistung. Wenn, wie es sich bei den Müllverbrennungsanlagen in Deutschland herausstellte, Landtagsabgeordnete „nebenbei" einen hochbezahlten Geschäftsführerposten bei einem Tochterunternehmen des privaten Betreibers, der Entsorgungsfirma Trienekens/RWE, erhalten (Rügemer 2002: 21), dann ist dies nicht strafbar.

Dasselbe gilt für die im Zuge der neoliberalen Umgestaltung der Kapitalgesellschaften ausgeweiteten Formen der Selbstbereicherung des Topmanagements. Neben das reguläre und publizitätspflichtige Gehalt treten immer mehr „Nebenleistungen", die inzwischen unter der Hand zu den Haupteinkommen mutiert sind: Aktienoptionen, Sonderprämien, Halteprämien, kostenlose Dienstwohnungen, Dienstwagen und Handys, Mitgliedsbeiträge für Golf- und Rotaryklubs, Pensionszuzahlungen, ausufernde Abfindungen beim Wechsel zu einer anderen Firma („Goldener Handschlag“). Die meisten dieser Vorteile sind an keine Leistung gebunden und werden vielfach auch bei schlechten Leistungen oder gar Versagen eingefordert und gewährt (Rügemer 2002a: 35f).

Exzessive Formen hat diese leistungslose, gegenseitige Selbstbevorteilung des Topmanagements bei Unternehmensfusionen angenommen: Die Zustimmung des Topmanagements des Übernahmekandidaten wird durch umfangreiche Zahlungen erkauft. Sie sind selbst zu einer wesentlichen Triebfeder für Unternehmensfusionen geworden (Blomert 2003: 51ff). Bei der Übernahme der Mannesmann AG durch den englischen Konzern Vodafone wurde die $\mathrm{Zu}$ - 
stimmung durch Zahlungen in der Gesamthöhe von 125 Millionen Euro an zwei Dutzend Mitglieder von Aufsichtsrat, Vorstand und weitere Topmanager befördert. Mannesmann-Aufsichtsratsmitglied Ackermann, zugleich Vorstandsvorsitzender der Deutschen Bank, der die Zahlungen mitbewilligt hatte, verteidigte sie mit der Begründung, sie seien üblich und im internationalen Vergleich ohnehin noch relativ niedrig (Süddeutsche Zeitung 18.2.2003). Mit dieser Feststellung hat der gewichtigste Bankier der Bundesrepublik Deutschland gewiss nicht unrecht. Es trifft auch zu, dass in keinem Staat der westlichen Wertegemeinschaft solche Zahlungen strafbar sind. Die deutschen Staatsanwälte, die aufgrund des politischen Protests in diesem herausgehobenen Fall (andere vergleichbare Fälle wurden justiziell nicht aufgegriffen) eine Anklage formulierten, mussten sich mit dem Gummi-Straftatbestand der „Untreue " behelfen. ${ }^{3}$

Die Formen der legalisierten Korruption sind unübersehbar geworden. Geheime Beraterverträge an politische Entscheidungsträger sind dabei noch eine vergleichsweise traditionelle Form. Zuletzt wurden drei Beraterverträge des gesundheitspolitischen Sprechers der CSU, Zimmermann, bekannt: er sollte erfolgsabhängig honoriert werden, wenn er für ein privates Krankenhausunternehmen staatliche Subventionen bei seinen Parteifreunden von der bayerischen Staatsregierung durchsetzen würde u.ä (Rügemer 2003). Unternehmen zahlen an Politiker für einstündige Standardreden problemlos Honorare zwischen 10.000 und 100.000 Euro. Die umstrittene Bank Clearstream in Luxemburg etwa zahlt an „key journalists" (Journalisten in strategisch wichtigen Medien) regelmäßige Beraterhonorare und hält sich den Außenminister des Landes ebenfalls mit einem Beratervertrag (Backes/Robert: 2003).

Solche Verpflichtungen und Abhängigkeiten werden zusätzlich verschleiert, wenn als Intermediär eine PR-Agentur auftritt. In Deutschland wurde neuerdings die Maklerfirma für politische Beziehungen Hunzinger AG auffällig. Sie finanziert Bücher von Politikern, vermittelt Politiker als Redner an Unternehmen und als Gesprächspartner an Manager, organisiert „Parlamentarische Abende“, vergibt günstige Privatkredite an Abgeordnete, richtete für den damaligen Verteidigungsminister Scharping ein Geldanlagekonto zu ungewöhnlich günstigen Bedingungen bei der einschlägig bekannten Privatbank Oppenheim ein, spendete selbst an alle Bundestagsparteien, vermarktete Ministerreisen in Krisengebiete wie den Kosovo. Zusätzlich unterhielt die Agentur ein politisches Meinungsforschungsinstitut und band auch Gewerkschaftsvertreter in Aufsichtsrat und Teilhaberschaften ein (Schwarz 1999: 253ff). So werden Politiker verschiedener Parteien, Topmanager und andere Mitglieder der Elite in ein undurchschaubares Geflecht eingebunden, das verdeckte Vorteile verschafft und verdeckte Abhängigkeiten erzeugt, die mit dem bisherigen

$3 \$ 266$ des Strafgesetzbuches. Vgl. zur Problematik des diffusen Straftatbestandes „Untreue“ Volhard (2002: 673ff). 
Recht - und auch mit den parlamentarischen Kontrollregularien - nicht erfasst werden.

\section{Globale Korruption nach 1945: Die stilbildende Kraft der USA}

Die Vereinigten Staaten von Amerika waren nach dem 2. Weltkrieg nicht nur die Führungskraft des damaligen Typs der neu einsetzenden Globalisierung, sondern auch der mit ihr organisch verbundenen Korruption. Schon damals waren die politisch-wirtschaftlichen Eliten der USA auch in dieser Hinsicht stilbildend.

Die Regierungskrise nach dem Rücktritt des Präsidenten Nixon 1975 ließ manche langgehegten korruptiven Seilschaften im In- und Ausland zerbrechen. Dies erwies sich als günstige Voraussetzung für den bisher größten Einblick in die globale Korruption. Zunächst untersuchten Kongressausschüsse das Verhalten des damals weltgrößten Rüstungslieferanten, Lockheed. Das Silicon-Valley-Unternehmen gab zu, allein in den Jahren 1970 bis 1975 insgesamt 202 Millionen Dollar an kickbacks (Rückvergütungen), Provisionen, Ausgleichszahlungen oder auch „unklaren Zahlungen“ an mehrere hundert Empfänger weltweit geleistet zu haben, um an Aufträge für Kampfjets und anderes militärisches Gerät zu kommen. $\mathrm{Zu}$ den Empfängern zählten Prinz Bernhard der Niederlande, der japanische Ministerpräsident Tanaka, der saudische Luftwaffenchef Hashim und der italienische Ministerpräsident Rumor. Solche Zahlungen leistete Lockheed seit Beginn der 50er Jahre, auch in der Bundesrepublik Deutschland, um dessen Wiederbewaffnung es damals ging (Boulton 1979).

Der „Lockheed-Schock“ führte dazu, dass die Geschäftspraktiken von weiteren 360 US-Firmen durchleuchtet wurden. Ihnen wurde Korruption nachgewiesen, insbesondere in "freundschaftlich verbundenen" Staaten. Die Bestecher trugen die renommiertesten Namen: Lockheed-Konkurrent Northrop, die Ölkonzerne Exxon, Mobil und Gulf, Nahrungsmittelkonzerne wie United Brands, Chemiekonzerne wie ICI, das Pharmaunternehmen Merck, Westinghouse, Firestone, Philipp Morris, ITT, Boeing. Gabuns Präsident Bongo (er kassierte auch von Elf-Aquitaine), Südkoreas Präsident Park, Boliviens Präsident Arrientos, der iranische Schah Pahlevi, der philippinische Präsident Marcos, der haitianische Präsident Duvalier gehörten zu den hochrangigen Empfängern, ebenso Tausende Minister, Generäle, Abgeordnete und Beamte aus der Türkei, aus Israel, Großbritannien, Guatemala, Deutschland, Indonesien, Argentinien, Kanada usw. Allein in Italien hatte Exxon teilweise direkt, teilweise über die Tochterfirma Esso Italia insgesamt 73 Millionen Dollar an Politiker der Regierungsparteien gezahlt (Sobel 1977). Es ging um konkrete Aufträge und Lizenzen, aber auch um die Stabilisierung eines politischen Umfelds, das für Superprofite US-amerikanischer Firmen günstig bleiben sollte. Dieses Umfeld 
war konservativ-christlich, schloss aber Diktaturen, Monarchien und rechtsradikale Gruppierungen ein, nach Bedarf und nationaler Kultur auch solche Kräfte wie die Mafia, luxemburgische Freimaurerlogen und kriminelle Geheimbünde aus der japanischen Unterwelt (Boulton 1979: 71ff, 195ff).

Von US-Unternehmen wurde auf diese Weise seit 1945 eine Struktur globaler Korruption praktiziert, die stilbildend bisherige Bakschisch- und Bargeldpraktiken überlagerte und schrittweise vereinheitlichte. Die Preise für die Auftragsbeschaffung und politische Systemsicherung stiegen enorm. Es etablierte sich der Berufsstand des „unabhängigen“ Provisions-Vermittlers (consultant). Schwarze Kassen und Briefkastenfirmen mit Vorratshaltung für Korruptionsgelder gehören seitdem zur Grundausstattung eines global player. Zudem wurde Korruption wissenschaftlich als Mittel der globalen Marktexpansion und der Förderung des US-Handels legitimiert. So schrieb der Politikwissenschaftler Samuel Huntington, die Korruption sei umso leichter akzeptierbar als sie die einzige Alternative zur Anwendung von Gewalt bei der Eroberung von Märkten darstelle und auch solchen Akteuren zu Einfluss verhelfen könne, die nicht über legitime institutionelle Kanäle verfügen (Huntington 1968; vgl. auch Nye 1967, Neff 1964).

Die Firmen im „Lockheed-Skandal“ waren auch die großen Zahler für die geheime Parteien- und Wahlkampffinanzierung in den USA selbst. Sie war damals verschleiert durch die Organisationsform der Political Action Committees (PAC): formal unabhängig, sammelten sie bei Unternehmen und leiteten die Gelder gezielt an bestimmte Kandidaten oder bei bestimmten Gesetzesvorhaben an die „richtigen" Empfänger. In diesem Zusammenhang stellte man fest, dass auch der Lobbyismus in Washington seine Gestalt geändert hatte. Die gesetzlich vorgeschriebene Registrierung der Lobbyisten wurde vor allem dadurch umgangen, dass große Rechtsanwaltsbüros die Lobbyarbeit übernahmen, da sie nicht als Lobbyisten im Sinne des Gesetzes galten (Etzioni 1984).

Die Korruption nach dem Lockheed-Muster ist inzwischen mit Hilfe der herrschenden Doppelmoral und der medialen Vergessenheitsproduktion aus dem Gedächtnis der US-amerikanischen wie der Weltgemeinschaft verschwunden. Gestützt wurde dieses Ergebnis dadurch, dass es nach der öffentlichen Skandalisierung so gut wie keine gerichtlichen Verurteilungen gab. So konnte unter der Hand eine neue Gestalt der globalen Korruption sich entwickeln, die freilich keineswegs so neu ist wie sie dem Publikum erscheint bzw. vorgespielt wird.

\section{Korruption und New Economy}

Die New Economy wurde in den Populärmedien, aber auch in Politik und Wissenschaft als ein Aufschwung der Aktienkultur (shareholder value) dargestellt, der sich auf den Bereich der neuen Kommunikationstechnologien und Medien einschließlich des Internet konzentriere. Für die wichtigsten Akteure, 
also die Investmentbanken, Wirtschaftsprüfer, Anwaltskanzleien, Personalberater, institutionellen Anleger, Analysten und Unternehmensvorstände und schließlich auch für die mit ihnen verbundene Politik ging es jedoch wesentlich um anderes.

Freigesetzt wurde die New Economy durch die tiefgehenden Deregulierungen, die mithilfe der republikanischen Mehrheit im US-Kongress ab 1994 durchgesetzt wurden (Scherrer 1999). Sie waren bereits unter den Präsidenten Reagan und George Bush vorbereitet worden. So wurde zunächst die Börsenaufsicht (Security Exchange Comission), die nach dem "Schwarzen Freitag" vom Oktober 1928 eingerichtet worden war und jahrzehntelang als die beste der Welt galt, in entscheidenden Teilen außer Kraft gesetzt. Die Trennung von Investment-, Kredit- und Aktienbanken wurde aufgehoben. Die Haftung für Banken, Anwaltskanzleien und Unternehmensvorstände wurde auf ein Minimum eingeschränkt. Die Selbstbedienung des Topmanagements bei der direkten Gewinnentnahme wurde ermöglicht. Die Nutzung von Finanzoasen wurde freigegeben. Mithilfe „kreativer“ oder ,aggressiver" Buchführung wurden Methoden der Bilanz- und Umsatzaufblähung kreiert, ebenso betrugsoffene Finanzprodukte wie Hedge-Fonds und Derivate. Die staatliche Kontrolle und Dokumentation insbesondere von grenzüberschreitenden Finanztransaktionen wurde weitgehend abgeschafft (Rügemer 2002b und c).

Diese Entwicklung war nur möglich, weil die wichtigsten Akteure auch die politischen Rahmenbedingungen beeinflussten. Dies lässt sich am politischen Weg des gegenwärtigen US-Präsidenten George W. Bush darstellen. Der wesentliche Sponsor seines Aufstiegs zunächst zum texanischen Gouverneur und dann zum US-Präsidenten war das Unternehmen Enron. Enron mit seiner Zentrale in Houston begann seinen Aufstieg zum siebtgrößten USUnternehmen ebenfalls in Texas. Es finanzierte nicht nur Bush, sondern auch die republikanische Partei. Ideologisch offen zahlte Enron auch an die demokratische Partei. Enron bedachte während der 90er Jahre 19 der 23 Mitglieder der Senatskommission für Energie im US-Kongress mit Spenden. Ebenso parteiübergreifend spendete Enron an Mitglieder weiterer Kommissionen. Zwei Drittel der US-Senatoren standen auf der Spendenliste des Konzerns. Desgleichen tat das Wirtschaftsprüfungsunternehmen von Enron, Andersen. Andersen spendete in gleicher Weise. Enron und Enron-Topmanager brachten allein für den Wahlkampf Bushs im Jahre 2000 insgesamt 1,96 Millionen Dollar auf. Die Firmenjets transportierten Bush zu Wahlkampfauftritten. Enron-Chef Kenneth Lay sponserte mit 100.000 Dollar die Präsidentschafts-EröffnungsGala im Januar 2001 (Hatfield 2002).

Als Gegenleistung setzte die Parlamentsmehrheit bisherige staatliche Kontrollen außer Kraft, zunächst in Texas, dann während der 90er Jahre in Washington. Dies betraf neben den obengenannten Bereichen insbesondere die Energie- und Finanzwirtschaft. So stieg Enron zum größten Händler von Gas- und Stromderi- 
vaten der Welt auf. Enron brauchte schließlich die Regierung nicht mehr zu bestechen, sondern stellt einen Teil der gegenwärtigen Regierungsmannschaft. Eine straflose neue Form der Korruption besteht in der Zahlung an Topmanager, damit sie einer Fusion zustimmen. So leistete Enron 76 Managern des Konkurrenten Dynegy, der im Jahre 2001 aufgekauft werden sollte, „retention bonus payments“. Sie betrugen je nach Stellung in der Unternehmenshierarchie zwrischen 75.000 und 1.800.000 Dollar. Sie wurden verdeckt über einen Trust gezahlt, der von der Wachovia Bank treuhänderisch gehalten wurde. Die Gesamtsumme der Zahlungen betrug 45.325.000 Dollar (Find Law, Enron Corp., Case No. 01.16034, Exhibit 3a.2).

Enron war mit denselben Methoden wie in Texas und den USA auch in weiteren Staaten tätig. Unter der Privatisierungs-Fundamentalistin Margret Thatcher konnte Enron zu Schleuderpreisen staatliche Kraftwerke in England aufkaufen und mit hohen staatlichen Subventionen neue Gaskraftwerke bauen. Die Europa-Zentrale von Enron wurde in London errichtet. Im Wahlkampf protestierte ein gewisser Tony Blair gegen das Eindringen Enrons. Er versprach den englischen Kumpeln, er werde für den Erhalt der heimischen Kohleförderung kämpfen. Im August 1998 sponserte Enron ein Galadinner beim Labour-Parteitag. Tony Blair ersetzte nach der Wahl im Energieprogramm seiner Regierung die Kohle durch Gas.

Im Jahre 2000 stieg Blairs Industrieminister Byers zum Chef von Enron Europe auf. Enron-Wirtschaftsprüfer Andersen wurde auch in England aktiv und sponserte ebenfalls Labour-Parteikonferenzen. An Schatzkanzler Brown verkaufte Andersen eine Luxuswohnung, die mindestens 350.000 Pfund Sterling wert war, für 130.000 Pfund Sterling. Auf Initiative Andersens legte Brown einen Gesetzentwurf zur Senkung der Gewinnsteuern vor - in den USA hatte Andersen es geschafft, dass Enron keine Steuern zahlte. Andersen entwickelte für die Blair-Regierung das Konzept Private Finance Initiative (PFI). Es besteht aus Buchführungs- und Haushaltstechniken, wie sie für Enron und andere Großunternehmen in den USA entwickelt worden waren. Sie erlauben, Schulden zu verstecken und finanzielle Ausgeglichenheit vorzuspiegeln. PFI soll auch vom englischen Staat genutzt werden (The Business 3./4. 2. 2002).

Im indischen Dabhol errichtete Enron für drei Milliarden Dollar ein GasKraftwerk. Kurz vor der Genehmigung im Jahre 1966 durch die zögernden indischen Behörden überwies Enron 100.000 Dollar an die demokratische Partei von Präsident Clinton, damit der US-Botschafter in Neu Delhi Druck mache. Nachdem sich das Projekt als überdimensioniert erwies und in Dabhol sich vehemente Proteste in der Bevölkerung wegen bevorstehender Strompreiserhöhungen häuften, verlangte Enron Subventionen. Im Mai 2000 wurde plötzlich ein neuer Punkt in das Energieprogramm der Bush-Regierung 
aufgenommen: Förderung der Stromerzeugung in Indien. ${ }^{4}$

In Afghanistan finanzierte Enron mit Dutzenden Millionen Dollar die Taliban. Zusammen mit dem Ölkonzern Unocal lud Enron 1997 und 1998 Taliban-Vertreter zu Verhandlungen nach Texas ein. Enron und Unocal wollten eine Pipeline durch Afghanistan legen, um Gas und Öl aus Turkmenistan und Usbekistan zu transportieren. Enron war bereit, den Taliban eine „Steuer“ auf jeden Kubikfuß Gas zu bezahlen, der durch die Pipeline fließen würde. „Enron würde auch mit dem Teufel paktieren, wenn es dem Unternehmen Gewinn bringen würde", kommentierte ein Mitglied des Kongressausschusses, der ab Januar 2001 den Enron-Konkurs untersuchte (The Enquirer 8.3.2002). Enron sponserte 1993 die Siegestour von George Bush nach dem ersten Golfkrieg durch Kuwait und wurde mit Aufträgen für den Wiederaufbau belohnt. Enron-Chef Lay hatte unmittelbaren Einfluss auf die Bestellung des Vorsitzenden der Federal Energy Regulatory Commssion und auf die Auswechslung eines missliebigen Kandidaten, indem nur der von Enron bevorzugte Konkurrent finanzielle Wahlkampfhilfe erhielt. Aufgrund der mithilfe von Strom- und Gas-Derivaten aufgeblähten Bilanzen stieg der Kurs der Enron-Aktien in bisher unbekannte Höhen. Enron verursachte die kalifornische Stromkrise im Winter 2000 und verdiente an der selbst hergestellten Verknappung des Stroms.

Die besondere Form der legalen Korruption manifestiert sich in der unmittelbaren Gewinnbeteiligung von Politikern an den von ihnen selbst herbeigeführten Projekten. Dick Cheney war bei George Bush bis 1992 Verteidigungsminister, unter dem Sohn George W. Bush ist er Vizepräsident. Die acht Jahre dazwischen überbrückte er als Präsident der Halliburton Industries, einem großen Zulieferer der Ölindustrie, der auch Geschäfte mit der irakischen Regierung von Saddam Hussein machte. Halliburton ist einer der großen $\mathrm{Zu}$ lieferer der US-Armee. Halliburton erhielt ohne Ausschreibung vor dem zweiten Golfkrieg Aufträge für den Wiederaufbau von Anlagen im Irak, deren Zerstörung von Cheney als Politiker mitbeschlossen wurden.

Richard Perle war stellvertretender Verteidigungs-Staatssekretär in der Regierung von Ronald Reagan. 1983 war bekannt geworden, dass er von einer israelischen Firma 50.000 Dollar erhalten hatte, um sich beim eigenen Ministerium für den Kauf von Waffen dieser Firma einzusetzen. Perle, langjähriger Befürworter des Kriegs gegen den Irak, ist eng mit der rechtsradikalen israelischen Likud-Partei verbunden. Bis Ende März 2003 war er Vorsitzender des Defense Policy Board, des US-Verteidigungsrates. Perle trat zurück, nachdem ein geheimer Beratervertrag mit Global Crossing bekannt wurde. 200.000 Dollar wurden ihm bereits ausgezahlt, eine Prämie von 750.000 Dollar sollte fol-

4 Vgl. Vallette/Wysham (2002: 31). Hier wird auch die Einflussnahme von Enron auf die Weltbank dargestellt. 
gen, wenn er den Widerstand des Pentagon brechen und für Global Crossing die Erlaubnis herausholen würde, ein sicherheitsrelevantes Glasfasernetz nach China zu verkaufen. Nach Perles Rücktritt wurde weiter bekannt, dass er an Trireme Partners beteiligt ist. Trireme investiert in Firmen, die Güter und Dienstleistungen für die US-Heimatverteidigung und die innere Sicherheit anbieten. Trireme wurde nach dem Attentat auf das World Trade Center im November 2001 gegründet und hat seinen Sitz in der inneramerikanischen Finanzoase Delaware (Hersh 2003, Steinberg 2003). So verdient der Unternehmer Perle an der Angst vor dem Terrorismus, die er als Politiker schürt.

Dies sind nur punktuelle Einblicke in eine neue Dimension der Korruption im Führungsstaat der, ,westlichen Wertegemeinschaft". Die Akteure haben sich im Vorausblick durch die Deregulierungen der 90er Jahre selbst als dauerhaft unschuldig erklärt. Alles, was sie tun, oder fast alles, ist „legal“. „Die US-Finanzindustrie steht vor ihrem moralischen Offenbarungseid. Juristisch aber hat sie wenig zu befürchten." (Der Spiegel 36/2002: 128) Von den frühen Insidergeschäften des jetzigen US-Präsidenten über die seines Vizepräsidenten bis zu denen des Managements von Enron \& Co: Sie sind nach geltendem Recht straflos. Wo noch ein Gesetz bestehen sollte, wird durch die Besetzung der Richterstellen nach dem republikanischen Parteibuch vorgebeugt: Am Ende der Gouverneurszeit von Bush 1999 in Texas waren alle 59 Bezirksrichter des Staates Republikaner. Auch die obersten Gerichte der USA sind in dieser Richtung umbesetzt und ermöglichten bekanntlich die „Wahl" des gegenwärtigen Präsidenten trotz ungenügender Stimmen (Hatfield 2002: 383).

Die Gewinnraten und Provisionen, die in den Topetagen der New Economy herausgeholt wurden, können selbst in einer deregulierten kapitalistischen Marktwirtschaft nicht auf Dauer „erwirtschaftet" werden. Auch die „friedliche" Korruption à la Huntington reicht schließlich nicht. Die Konkurse von Enron, Worldcom usw. zeigen es. Die „friedliche" Selbstbereicherung geht organisch, wenn nötig, auch zu militärischen Mitteln über. Huntington, der 1968, als die USA mit der Sowjetunion noch einen militärischen Gegenpart hatten, die Korruption als legitimes Instrument der Ausweitung des freien Marktes und insbesondere des US-Handels gerechtfertigt hatte, weil sie die bessere Alternative zur Gewaltanwendung sei, hat seine Theorie bekanntlich unter den veränderten Umständen weiterentwickelt und die gewaltsame $\mathrm{Zu}$ spitzung der internationalen Situation im "clash of civilisations" begründet (Huntington 1996).

Als Sinnbild für die organische Verbindung von Marktausweitung, Korruption und militärischer Gewalt kann das Vorgehen der US-Regierung in Afghanistan gelten. Die geschilderten Interessen von Enron, Unocal usw. waren mit den Taliban nicht durchzusetzen. Zur Vorbereitung des militärischen Eingreifens gab die CIA 70 Millionen Dollar für den Kauf von Taliban-Warlords aus. Mittlere Kommandeure waren mit Summen bis zu 50.000 Dollar zu haben, 
künftige Minister der neuen Regierung mit bis zu einer Million. Bei der militärischen Intervention winkten die US-Offiziere dann mit Dollarbündeln, um Überläufer zu kaufen. Präsident Bush war über die niedrige Summe erfreut, die man hatte einsetzen müssen. „Das ist ein gutes Geschäft“, wird er zitiert (Woodward 2002).

\section{Alle kämpfen gegen die Korruption?}

Die wichtigste Maßnahme - zumindest auf Regierungsebene - gegen die globale Korruption war zunächst der Foreign Corrupt Practices Act (FCPA) von 1977. Dieses Gesetz zur Bekämpfung der Bestechung durch US-Unternehmen im Ausland war die Antwort des US-Kongresses auf den "Lockheed-Skandal“" In den Jahren danach wurden ungefähr ein halbes hundert US-Unternehmen wegen bekannt gewordener Bestechung - unter anderem in Deutschland, Israel und Ägypten - bestraft, darunter wiederholt auch Lockheed (Rügemer 1996: 128ff). Da die USA mit ihrem Gesetz aber alleine blieben, wurde es abgeschwächt und mit Ausnahmen versehen. US-Wirtschaftsminister Brown legte schließlich 1995 eine Studie vor, in der die Benachteiligung der US-Firmen gegenüber ihren Konkurrenten wie Siemens, Alstom und Airbus dargestellt wurde, die straflos an Bestechungspraktiken festhalten konnten (Judge 1995). Dies war der Anlass für eine zweite wichtige Maßnahme: Die in der OECD vertretenen 29 Industriestaaten einigten sich 1997 auf zwei Konventionen: 1. Verbot der steuerlichen Begünstigung von Schmiergeldern und 2. Strafbarkeit der Bestechung ausländischer Amtsträger (vgl. www.oecd.org/daf/nocorruption). Die Konventionen wurden in den meisten Mitgliedsstaaten in nationales Recht umgesetzt, auch in Deutschland, ${ }^{5}$ nicht aber in den Finanzoasen Schweiz und Luxemburg. Freilich ist zum Beispiel in Deutschland von der Umsetzung in den Unternehmen, Finanzämtern, Staatsanwaltschaften und Gerichten nicht viel zu spüren. Die neuen Gesetze werden von Staat wie Unternehmen im wesentlichen als Pflichtübung angesehen. Die Bevölkerung hat kaum Kenntnis ron der neucn Rechtslage. So ist beispiclsweise noch kein Fall bekannt geworden, dass in Deutschland, Frankreich, Großbritannien usw. ein einheimisches Unternehmen wegen Bestechung im Ausland angeklagt wurde.

Die neue Gesetzgebung hat dagegen eine Konjunktur "neuer Ethiken" hervorgerufen. Zahlreiche Vereinigungen und Lehrstühle für "Wirtschaftsethilk" wurden eingerichtet. Viele Konzerne haben „,codes of conduct", also unternehmensinterne Verhaltensregeln, verabschiedet. Dies sind Selbstverpflichtungen, die von niemandem als den Betroffenen selbst überprüft werden. Die Formulierungen sind abstrakt und diffus. Zudem läge die ethische Lösung des Problems nicht darin, dass gesonderte Wirtschafts- und Unternehmensethiken

5 Vgl. Abzugsverbot für die Zuwendung von Vorteilen im Sinne des $§ 4$, Absatz 5, Satz 1 Nr. 10 Einkommensteuergesetz, in: Bundessteuerblatt 2002, Teil I, 10.10.2002, S. 1031. 
produziert werden, sondern dass die Wirtschaftsalkteure sich der Ethik nach den sonst geltenden demokratischen Standards unterwerfen und keine Sonderrolle beanspruchen.

Die Flut neuer Wirtschaftsethiken wird beispielsweise durch den ehemaligen Präsidenten des Bundesverbandes der Deutschen Industrie, Hans-Olaf Henkel, genährt. Er bezeichnet die Korruption als „einen der fleißigsten Totengräber der Freiheit" (Henkel 2002: 225). Er plädiert zwar einerseits dafür, dass neben den Bestochenen auch die Bestecher bestraft werden. Er wettert aber dann nur gegen „die Führungsschicht der armen Länder, die durch ihre Bestechungsanfälligkeit ihre Völker im Elend halten", denn diese Führungsschicht würde „,ihren Bürgern mehr Schaden zufügen als je ein realer oder eingebildeter Großkapitalist" (ebd.: 226). Dass das Schmiergeld für die „Anfälligen" vom zweiten Beteiligten der korruptiven Gemeinschaft kommt, also jenem „Großkapitalisten", und dass dieser auch im eigenen reichen Staat Politiker und Beamte besticht und auch hier die Bürger schädigt - das blendet Henkel aus. Er rettet das alte Klischee, und die Schmiergeldzahler der Industriestaaten sind wieder schuldlos gestellt.

Henkel beruft sich bei seiner Polemik gegen die Führungsschicht der armen Länder auf den „Korruptionsindex" der Anti-Korruptionsorganisation Transparency International (TI). TI wurde 1993 von einem ehemaligen Weltbankdirektor gegründet und besteht inzwischen mit nationalen Sektionen in über 80 Staaten (vgl. www.transparency.org). Das zeugt von der schnell wachsenden Sensibilität gegenüber dem lange tabuisierten Problem der Korruption. TI ist mit dem Anspruch angetreten, das jahrzehntelang vorherrschende Klischee von den „korrupten Entwicklungsländern“ zu überwinden. TI vor allem ist es zu verdanken, dass die OECD die beiden genannten Konventionen verabschiedet und in den wichtigsten Industriestaaten eine Welle von AntiKorruptions-Gesetzen in gang gesetzt hat. TI hat in weiteren internationalen Gremien wie der UNO, der Weltbank, dem Europarat und der EU einen geachteten Konsultativstatus erworben. Dabei half die prominente TIMitgliedschaft ehemaliger Staatsoberhäupter, Generalstaatsanwälte und Unternehmenschefs. Freilich ist mit diesem Aufstieg eine Entwicklung zur Unglaubwürdigkeit verbunden. Darin spiegelt sich ein Dilemma, dem gewiss nur schwer zu entkommen ist.

TI geht von der zutreffenden Annahme aus, dass der entscheidende Hebel zur Bekämpfung der Korruption in den Unternehmen liegt. Deshalb möchte TI den vertraulichen Dialog mit den Unternehmen und der Unternehmenslobby pflegen. Unternehmen werden als Fördermitglieder der Organisation gern gesehen. Das hat bereits in wenigen Jahren dazu geführt, dass unter der Hand eine weitgehende Interessenidentität entstanden ist, die den Zielen der Organisation widerspricht. Der jährlich veröffentlichte Korruptionsindex gibt die Perspektive der Bestecher wieder: er beruht auf einer Befragung westlicher Ge- 
schäftsleute, in welchen Staaten sie der Korruption begegnen. Die andere Seite wird nicht befragt.

TI hatte Enron bei seinem endgültigen Aufstieg zum Weltkonzern als Sponsor umworben. Enron trat als Fördermitglied bei und spendete in den Jahren 1995 bis 1999 jeweils 10.000 Dollar an die US-Sektion von TI (Mitteilung von TI-USA 24.3.2003). ${ }^{6}$ Ein großer Teil der korruptiven Spendenpraxis von Enron hinsichtlich des Bush-Clans und der politischen Landschaft in Washington war damals bereits bekannt, wurde aber von TI nicht kritisiert. Die Unglaubwürdigkeit beginnt jedoch spätestens dann, wenn das ganze Ausmaß der Korruption bekannt wird und dennoch unkommentiert bleibt. Dies war nach dem Konkurs von Enron Ende 2001 der Fall.

TI ging in der Öffentlichkeit nicht auf das Verhalten ihres wesentlichen Sponsors ein. Vielmehr gab die US-Sektion im Juli 2002 eine Erklärung heraus, in der lediglich bedauert wird, dass der ,Skandal" um Enron und dessen Wirtschaftsprüfer Andersen die "Glaubwürdigkeit der USA im Ausland" und die „Führerschaft der USA geschwächt“ habe. Auch die Bestechungspraktiken Enrons und der CIA in Afghanistan werden nicht thematisiert. Desgleichen gehört etwa der Erdölkonzern Unocal zu den Fördermitgliedern, ohne dass dessen Korruptionspraxis in Afghanistan von TI thematisiert würde. TI bindet sich in das provinzielle, nationalistische und diffuse Kriterium „Glaubwürdigkeit der USA" ein und verhält sich somit desaströs gegenüber den eigenen Zielen.

Als Konsequenz aus dem Enron-Skandal forderte TI, dass Unternehmen freiwillige Anti-Korruptions-Programme etablieren sollten (TI-USA 2002). Dass Enron selbst schon ein solches Programm eingerichtet hatte, und zwar mit Hilfe von TI, und als aufwendig gedrucktes Handbuch an die Mitarbeiter verteilen ließ - dies blieb unerwähnt. Als weitere Konsequenz forderte TI eine bessere Anwendung der General Accepted Accounting Principles (GAAP), also der in den USA geltenden Bilanzregeln. Doch will man etwas gegen Korruption in der Unternehmens- und Finanzwelt tun, dann müssen zunächst alle Deregulierungen zurückgenommen werden, die während der 90er Jahre unter dem Stichwort „kreative Buchführung" durch die Wirtschaftsprüfer in die GAAP eingeführt wurden. Sie sind die wesentliche rechtliche Ursache für die Bilanzmanipulationen bei Enron, Andersen \& Co. Zudem sind die Wirtschaftsprüfer in allen westlichen Staaten über die Schweigepflicht gesetzlich und finanziell so eng an das beauftragende Unternehmen gebunden, dass die buchhalterische und bilanzielle Mitgestaltung korruptiver Geldflüsse zu ihren Aufgaben gehört, z.B. auch die Gründung geeigneter Briefkastengesellschaften in Finanzoasen, wie bei Enron geschehen (Rügemer 2000).

6 Am 6.5.2003 teilte TI-USA auf Nachfrage nnit, dass auch Andersen von 1995 bis 2000 Fötdermitglied war und jährlich $10.000 \$$ spendete. 
Es gibt keine Korruption und keine größere Bilanzmanipulation der letzten Jahre, auch in Deutschland (vgl. die Fälle Holzmann, Flowtex, Comroad und viele andere), die nicht durch einen der „renommierten“ Wirtschaftsprüfer mitgestaltet und jeweils bis knapp vor dem Konkurs als ordnungsgemäß testiert worden wären. Dennoch gehören die führenden Unternehmen KPMG, Price Waterhouse Coopers und Ennst \& Young ohne jegliche Problematisierung zu den langjährigen Fördermitgliedern von TI (vgl. www.tiusa.org).

Eine ähnliche Selbsterblindung zeigt sich beim Problem der Geldwäsche. TI hat erkannt, dass Korruption und Geldwäsche eng miteinander verbunden sind. Deshalb traf TI im Oktober 2000 mit 12 führenden Großbanken eine Vereinbarung gegen Geldwäsche. Zu den in der "Wolfsberg-Gruppe" zusammengefassten Banken gehören ABN-AMRO, Banco Santander, Barclays, Citigroup, Credit Suisse Group, Deutsche Bank, Goldman Sachs, HSBC, JP Morgan Chase, Société Générale, Bank of Tokyo-Mitsubishi und UBS. Die Nutzung des Bankensystems für kriminelle Zwecke und für Terroristen soll unmöglich gemacht werden, Banken sollen nur solche Kunden akzeptieren, bei denen die Herkunft von Geld, Vermögen und sonstigen Werten ,vernünftigerweise als legitim betrachtet werden kann" (The Wolfsberg Anti-Money Laundering Principles vom 30.10.2000, in: www.wolfsberg-principles.com).

Deshalb ist in der Vereinbarung festgelegt, dass keine Beziehungen zu „Briefkastenbanken" aufgenommen werden sollen. Freilich wird eine Ausnahme gemacht, wenn die Briefkastenbank eine „regulierte Tochtergesellschaft" (regulated affiliate) einer regulären Bank ist. Dies kommt den großen Banken entgegen, die heute die offshore-Bankparadiese extensiv nutzen. So hat allein die Deutsche Bank ca. 350 Tochtergesellschaften auf den Cayman Islands (Deutsche Bank 2002). In der Vereinbarung wird weiter die sorgfältige Prüfung der „Korrespondenzbanken" festgelegt. Wenn eine Bank in einem anderen Staat keine eigene Filiale hat, kann sie zu einer dortigen Bank eine Korrespondenzbeziehung unterhalten und auf diese Weise Transfers abwickeln. Die AntiGeldwäsche-Kommission des US-Senats hat in umfangreichen Hearings und Gutachten dargestellt, wie schon durch Kreditkarten regulärer US-Banken der Zugang zu gewaschenen Geldern von Korrespondenzbanken möglich ist (vgl. Corespondent Banking Industry in the United States, Februar 2001, in: www.levin.senate.gov/issues/money). Dieses Problem wird in der „WolfsbergErklärung" ausgespart. Zudem ermöglichen es die internationalen ClearingInstitutionen für grenzüberschreitende Wertpapier-Transaktionen wie Clearstream (Luxemburg) und Euroclear (Brüssel), dass nichtregulierte Briefkastenbanken Beziehungen zu „regulären“ Banken und Unternehmen unterhalten. In den Verwaltungsräten von Clearstream und Euroclear sitzen aber neben anderen auch die Banken der Wolfsberg-Gruppe und brechen damit täglich ihre mit TI geschlossene Vereinbarung (Backes/Robert 2003).

Schließlich wird der heute entscheidende Teil der Geldwäsche von den Groß- 
banken selbst durchgeführt. Auch dies ist „legal“. Es geschieht vor allem mithilfe von Derivaten, die staatlich weder kontrolliert noch dokumentiert werden und auch keine personenbezogene Erfassung von Eigentümern erfordern wie sie in der Wolfsberg-Vereinbarung gefordert wird (Hafner 2002: 99ff). Die Verantwortlichen von TI kleben an einem anachronistischen Bild der Geldwäsche, das von Banken unterstützt wird. Ob es sich hinsichtlich der kritiklosen Haltung gegenüber den modernsten und entscheidenden Formen der Geldwäsche bei TI um banktechnische Inkompetenz, naives Vertrauen oder um bewusste Verschleierung handelt, ist nicht ersichtlich, letztlich auch unerheblich. Auch die Weltbank hat sich ein Anti-Korruptions-Programm gegeben. Eine konkrete Durchsetzung vor Ort ist allerdings kaum erkennbar. Erst wenn Nicht-Regierungsorganisationen aktiv werden, kommen in der Regel Aktivitäten gegen Korruption in gang. Dies zeigte sich zum Beispiel beim BujagaliStaudammprojekt in Uganda. Nach Weltbank-Präsident Wolfensohn handelt es sich um ein vorbildliches Modell für privatisierte Entwicklungshilfe. Das US-Unternehmen Applied Energy Systems (AES) will den Staudamm als Investor errichten und den hier erzeugten Strom 30 Jahre lang zu festgelegten Preisen und Kontingenten an den Staat verkaufen. Der verschuldete Staat Uganda müsste keine Kredite aufnehmen, aber in der ersten 12 Jahren jeweils 100 Millionen Dollar zahlen. Der Preis des Stroms würde bei 20 Cent pro Kilowatt liegen statt wie bisher bei 8 Cent. Teilhaber in dem mit der Regierung gegründeten Unternehmen AES Nilepower sind auch ein Bruder und eine Schwester von Präsident Museweni. Der Standort wurde auch deshalb ausgesucht, weil der Bruder dort ein Grundstück einbrachte. Es gab keine öffentliche Ausschreibung. Der Vorstandsvorsitzende von AES gehört der religiösen Gruppierung „Wiedergeborene Christen" an, ebenso wie Museweni. Nachdem das Parlament von Uganda jahrelang über dieses größte nationale Projekt diskutiert hatte und Bestechungsvorwürfe aufkamen, beschloss es in einer Dringlichkeitssitzung im Sommer 1999 bei Anwesenheit nur eines Drittels der Abgeordneten den Vertrag mit AES. Er blieb bis heute geheim.?

Dies sind typische Indizien für Korruption. Organisationen wie die „Erklärung von Bern" und „International Rivers Network" haben das Projekt vor Ort begleitet und frühzeitig Kritik geäußert. Die ugandische Anwaltsgruppe „Green lawyers" kämpfte seit dem Parlamentsbeschluss um die Veröffentlichung des Vertrags, die schließlich gerichtlich erzwungen wurde. Trotz ihres Anti-Korruptionsprogramms genehmigte die Weltbank jedoch dem Unternehmen AES die für den Bau beantragten Kredite.

Die Schweizer Regierung nahm dies zur Grundlage für den Beschluss, dem Unternehmen Alstom für dessen Projektanteil (Turbinen) eine ExportRisikogarantie zu geben, übrigens mit der Begründung, dass eine Auftragsver-

7 Vgl. die detaillierte Darstellung durch die Organisation „Erklärung von Bern“ in: www.evb.ch 
gabe ohne öffentliche Ausschreibung in Entwicklungsländern ,üblich“ und deshalb nicht zu beanstanden sei. Die Weltbank hält ihre eigene Untersuchung über den wirtschaftlichen Nutzen des Projekts unter Verschluss. Erst als bekannt wurde, dass das norwegische Unternehmen Veidekke, das am AES Nilepower-Konsortium beteiligt ist, über ihre englische Tochterfirma einen ugandische Beamten bei einem kleineren Staudammprojekt bestochen hatte, blockierte die Weltbank im Jahre 2002 ihre Entscheidung.

Staaten und internationale Organisationen wie die Weltbank neigen trotz neuer Anti-Korruptionsprogramme in der Praxis dazu, sogar die (noch) strafbare Korruption zu decken. Um die legalisierte Korruption kümmern sie sich nicht. Die wichtigste Anti-Korruptions-Organisation TI ist in kurzer Zeit bei Regierungen und internationalen Organisationen zu einem anerkannten Akteur geworden, ist aber dabei, Glaubwürdigkeit und Kompetenz zu verlieren. Dies ist bei einer weitgehend als Honoratiorenclub organisierten Gruppe im Umgang mit den aktuell Mächtigen nicht grundsätzlich überraschend, belegt aber die Richtigkeit der Aussage, mit der TI angetreten ist: die staatlichen und privaten Akteure, die die gegenwärtige internationale Weltordnung einschließlich der systemischen Korruption aufgebaut haben, können sich nicht selbst daraus befreien. Die "Zivilgesellschaft", die zurecht als notwendiger Gegenspieler beschworen wird (Eigen 1999), muss offensichtlich sehr viel tiefer ansetzen, nicht nur im Bereich der Korruption.

\section{Literatur}

Aburish, Said K. (1995): The Rise, Corruption and coming Fall of the House of Saud. New York Backes, Ernest; Robert, Denis (2003): Das Schweigen des Geldes. Die Clearstream-Affare. Zürich

Bannenberg, Britta (2002): Korruption in Deutschland. Neuwied

Blomert, Reinhard (2003): Die Habgierigen. Kapitalismus außer Kontrolle. München

Boulton, David (1979): Die Lockheed-Papiere. Politik und Geschäft der Rüstungsgiganten. Oldenburg/München

Calvi, Fabrizio; Sisti, Leo (1995): Les Nouveaux Réseaux de la corruption, Paris

Carini, Marco (2002): Vergoldeter Müll, in: Freitag 28.3.2002

Deutsche Bank (2002): Verzeichnis des Anteilsbesitzes 2001, Frankfurt/Main

Eigen, Peter (1999): Vorwort, in: Pieth/Eigen

Etzioni, Amitai (1984): Capital Corruption. The New Attack on American Democracy. New York/London

George1, Jacques; Thorel, Anne-Marie (1997): Dictionnaire des „Affaires“. Argent et Politique, Paris

Gherardo Colombo (1995): Korruption als Flächenbrand, in: Friedrich-Ebert-Stiftung (Hrsg.): Korruption in Deutschland, Berlin

Hafner, Wolfgang (2002): Im Schatten der Derivate. Das schmutzige Geschäft der Finanzelite mit der Geldwäsche. Frankfurt

Hatfield, James (2002): Das Bush-Imperium. Bremen

Henkel, Hans-Olaf (2002): Die Ethik des Erfolgs. Spielregeln für die globalisierte Gesellschaft. München

Hersh, Seymour (2003): Annals of National Security: Lunch with the Chairman, in: The New Yorker 10.3.2003;

Huntington, Samuel (1968): Political Order in Changing Societies, Yale Universität Press

Huntington, Samuel (1996): The Clash of Civilizations. New York 
Jacoby, Neil; Nehemkis, Peter; Eells, Richard (1977): Bribery and Extortion in World Business. New York

Judge, Paul (1995): A World of Greased Palms - Inside the Dirty War for Global Business, in: Business Week 6.1.1995, S.30f.

Kilz, Hans-Wemer (1983): Die gekaufte Republik, Hamburg

Leyendecker, Hans u.a. (2000): Helmut Kohl, die Macht und das Geld, Göttingen

Neff, Natanael (1964): Economic Development through Bureaucratic Corruption, in: American Behavioral Scientist, November, S. 8ff.

Nye, Joseph (1967): Corruption and political development - a Cost-Benefit Analysis, in: American Political Review 2/1967, S. 417ff.

Pieth, Mark; Eigen, Peter (Hg.) (1999): Korruption im internationalen Geschäftsverkehr. Neuwied/Basel

Queloz, Nicolas u.a. (2000): Processus de corruption en Suisse, Basel/Genf/München

Raffy, Serge (1996): Corruption - L'affaire Bouygues, in: Le Nouvel Observateur 25.1.1996

Rodolf Heußner (2002): Ich, der Sündenbock. Bad Neustadt

Rose, Matthew (2003): Eine ehrenwerte Gesellschaft. Die Bankgesellschaft Berlin. Berlin;

Rügemer, Werner (1995): Staatsgeheimnis Abwasser. Düsseldorf

Rügemer, Werner (1996): Wirtschaften ohne Korruption? Frankfurt/Main

Rügemer, Werner (2000): Geldwäsche via Internet. Finanzoasen rüsten auf, in: Freitag 12.5.2000

Rügemer, Werner (2002): Colonia Corrupta. Münster;

Rügemer, Werner (2002a): arm und reich, Bielefeld

Rügemer, Werner (2002b): Die große Aufblähung. Warum die US-Wirtschaft größer scheint als sie ist, in: Kommune 8/2002;

Rügemer, Wenner (2002c): Riskante Peanuts aus der Karibik. Europäische Städte schließen dubiose US-Leasing-Verträge ab, in: Kommune 2/2002

Rügemer, Werner (2003): Wieviele Beraterverträge? Porträt eines führenden Gesundheitspolitikers, in: www.verdi.de/Gesundheitsreform März 2003

Scherrer, Christoph (1999): Globalisierung wider Willen? Die Durchsetzung liberaler Außenwirtschaftspolitik in den USA. Berlin

Schwarz, Friedhelm (1999): Das gekaufte Parlament. Die Lobby und ihr Bundestag. München/Zürich

Sobel, Lester (1977): Corruption in Business, New York

Steinberg, Michele (2003): Cheney and Perle to go down like Ollie North?, in: Executive Intelligence Review 21.3.2003

TL-USA (2002): Statement on Corporate Governance and Accounting Reforms, Washington

Vallette, Jim; Wysham, Daphne (2002): Enron's Pawns. How Public Institutions Bankrolled Enron's Globalization Games. Washington

Volhard, Rüdiger (2002): Die Untreuemode, in: Festschrift für Klaus Lüdersen, Baden-Baden

Woodward, Bob (2002): Bevor der Krieg gegen die Taliban begann, infiltrierte die CIA das Land, in: Die Welt 3.12 .2002 